\title{
Dietary intake of energy and selected nutrients and plasma lipid profile in young women and men with different levels of physical activity
}

\author{
Marzena Malara, Elżbieta Hübner-Woźniak \\ Department of Biochemistry and Biology, University of Physical Education in Warsaw, Poland
}

\section{Summary}

Study aim: The purpose was to assess dietary intake of energy and selected nutrients and plasma lipid profile in young women and men with different levels of physical activity.

Material and methods: The research included a total of 116 female and male students at the University of Physical Education who differed in their levels of physical activity. Analysis of the diets was based on 24-hour dietary interviews collected over 4 days prior to blood collection. Concentrations of total cholesterol (TC), HDL cholesterol (HDL-C), and triacylglycerol (TG) were measured in venous blood. In addition, the concentration of LDL cholesterol (LDL-C) and the ratios of TC / HDL-C and LDL-C/HDL-C were calculated.

Results: Women, regardless of their physical activity levels, were characterized by a significantly higher concentration of total cholesterol in plasma $(4.8$ and $4.5 \mathrm{mmol} / \mathrm{L}$, respectively - in training participants; 4.7 and $4.3 \mathrm{mmol} / \mathrm{L}$, respectively - in non-training participants), although their dietary intake of cholesterol was significantly lower in comparison with the corresponding groups of men (282.0 and $484.7 \mathrm{mg}$, respectively - in training participants; 252.6 and $400.2 \mathrm{mg}$, respectively - in non-training participants). A trend toward a worse lipid profile of training women and men compared with the corresponding groups of non-training participants was also observed.

Conclusions: Increased physical activity in the groups of training women and men was not a sufficient stimulus to induce positive changes in their lipid profiles. Nor can it be excluded that the observed differences were the result of diet, as dietary intake of cholesterol in the groups of training women and men was higher compared with the corresponding groups of non-training participants.

Keywords: Lipid profile - Dietary habits - Physical activity - Women - Men

\section{Introduction}

It is commonly known that factors such as gender, age, diet, physical activity, and substance abuse have an impact on the proper functioning of the body. The findings of numerous studies have indicated a close relationship between rational nutrition and regular physical activity, and the lower risk of atherosclerosis and its sequelae in the form of cardiovascular disease. These illnesses, related to disorders of lipids and lipoproteins, manifest in increased concentrations of triglycerides, total cholesterol, and LDL cholesterol, as well as decreased concentrations of plasma HDL cholesterol [36].

Unbalanced diet, as characterized by an excess of energy relative to demand as well as a high content of fat rich in saturated fatty acids, has been shown to contribute to disorders of lipid profile [5]. Jakobsen et al. [18] found that the risk of cardiovascular disease increased significantly in women whose dietary intake of saturated fats rose by $5 \%$. However, such a relationship was not observed among men. Similarly, Montoya [29] noted that in a group of people who for 4-5 weeks consumed a diet rich in saturated fats, the concentrations of TG, TC, and LDL-C in plasma increased in comparison with people who had a diet rich in monounsaturated fats and polyunsaturated fats for the same period, though the percentage of energy from fat in both types of diets was the same. High intake of cholesterol, usually associated with a diet containing an excess of saturated fats, and high intake of trans unsaturated fatty acids isomers adversely affect lipid profile equally $[3,23]$. However, dietary cholesterol is absorbed from the gastrointestinal tract at rates up to 25-30\%. The degree of its absorption depends mostly on the amount of bile secreted and, to a lesser extent, on fat and cholesterol levels in foods. Much of the data in the literature also indicate that reducing the supply 
of cholesterol in a diet and the factors that decrease intestinal cholesterol absorption only minimally contribute to lowering concentrations of plasma cholesterol [21]. Furthermore, it should be noted that cholesterol is constantly synthesized in the human body, and the initial substrate for production of this compound is acetyl-CoA. Endogenous synthesis of cholesterol is located mainly in the liver and depends in part on the degree of intestinal cholesterol re-absorption from bile, as well as the supply of cholesterol and saturated and unsaturated fatty acids in a diet [2]. Research findings also suggest that a high dietary intake of carbohydrates with a high glycemic index, such as sucrose and its component fructose, may contribute to the development of hypertriglyceridemia and insulin resistance, which increase the risk of cardiovascular disease [11,37]. Bantle et al. [1] observed a significant increase in the concentration of plasma triacylglycerol and lack of change in the concentrations of total cholesterol and its fractions in a group of men who for 6 weeks consumed a diet of which $17 \%$ was energy from fructose.

It should also be emphasized that a diet containing an adequate amount of unsaturated fatty acids has a positive effect on lipids and lipoproteins in the body. This was confirmed by Hodson [16] and Müller [30], who observed favorable changes in the lipid profiles of participants who replaced in their diets products rich in saturated fats with products rich in unsaturated fats.

Moreover, it has been demonstrated that even a moderately high intake of carbohydrates with a low glycemic index helps to lower the risk of cardiovascular disease. Vidon et al. [40] demonstrated both a significant decrease in the total cholesterol and LDL cholesterol concentrations as well as an increase in the concentration of HDL-C lipoprotein in their research involving people who for a period of 3 weeks consumed a diet characterized by a higher amounts of energy derived from carbohydrates and a lower amounts of energy from fat.

Favorable changes in a plasma lipid profile have also been observed as a result of increased consumption of fruits and vegetables that are a rich source of dietary fiber [33, 35]. Erkkila et al. [8] found that the total cholesterol concentration in plasma and the risk of cardiovascular disease were significantly and negatively correlated with the intake of dietary fiber.

Undoubtedly, regular physical activity has a beneficial impact on lipid profile, as active people have lower concentrations of triglycerides, total cholesterol, LDL cholesterol and higher concentrations of HDL cholesterol in plasma in comparison with inactive people [15].

At the same time, there have been no conclusive findings in regard to what intensity, frequency, and duration of exercise are sufficient to induce favorable changes in lipid profiles. Okura et al. [32] demonstrated that a decrease in LDL-C concentration was greater after high-intensity physical activity than after low-intensity activity. Yet Durstine et al. [7] have suggested that favorable changes in lipid profile observed as a result of exercise depend mostly on the duration of training and initial levels of lipid profile components.

In addition, data in the literature have shown that sex is an important factor in the regulation of lipids and lipoproteins in the body. Men's lipid profile has higher concentrations of both triglycerides and LDL cholesterol, and a lower concentration of HDL cholesterol in plasma, in comparison with women's lipid profile [10]. Female sex hormones are believed to be responsible for these differences because the hormonal changes caused by menopause promote adverse changes in lipid profile, which increases the risk of cardiovascular disease in a group of the women compared with men of the same age [14].

Taking into consideration the influence of the factors noted above on the metabolism of lipids and lipoproteins, the purpose of this study was to assess dietary intake of energy and selected nutrients and plasma lipid profile in young women and men with different levels of physical activity.

\section{Material and methods}

The study included a total of 116 female and male students at the University of Physical Education who differed in their levels of physical activity. Groups of non-training participants consisted of 30 women and 27 men (NW - non-training women; NM - non-training men) participating only in physical education classes that were part of their curriculum at the university. Groups of training participants consisted of 29 women and 30 men (TW - training women; TM - training men) who trained in various endurance sports in addition to their physical education classes at the university. Participants' levels of physical activity were evaluated on the basis of oral statements about their organized physical activity (for non-training groups - physical activity during physical education classes associated with the curriculum; for training groups - physical activity during physical education classes associated with the curriculum and the extracurricular training for endurance sports).

All participating women and men gave written consent to participate in the research, which started after obtaining the approval of the local Ethics Committee.

All participants were weighed and measured in order to calculate their BMI. Skinfold measurement were performed in a standing position, on the left side of the body, using the Holtain skinfold caliper (United 
Kingdom). Each measurement of the 4 skinfolds (above the arm triceps and biceps; below the shoulder blade; above the right anterior superior iliac spine) was made 3 times. Mean values were used to compute the percentage of body fat. The method of Durnin et al. [6] was applied in order to assess the percentage of body fat.

Dietary intake of energy, protein, fat, fatty acids, carbohydrates, and cholesterol were determined on the basis of 24-hour dietary interviews collected over 4 days prior to blood collection. Interviews took into account the menus of 2 work days and 2 days off (Saturday and Sunday). Daily food intake was assessed using the Photo Album of Products and Dishes, which presented the types and sizes of foods in grams [38], and then was analyzed by Dieta 4.0, a computer software developed by the National Food and Nutrition Institute in Warsaw. Dietary analysis, including the dietary intake of the aforementioned components, was performed according to guidelines provided by the Department of Sports Nutrition at the Australian Institute of Sport and by Lemon [4, 22]. The lipid profile was performed according to guidelines provided by the Third Report of the National Cholesterol Education Program (NCEP) Expert Panel (United States) [39].

Following an overnight fast, venous blood samples were collected in polypropylene tubes containing an anticoagulant and then centrifuged for 15 minutes (3000 rev/min) in order to obtain plasma that was later stored at $-70^{\circ} \mathrm{C}$ until the assays were performed.

Total cholesterol (TC) concentration was determined colorimetrically at a wavelength of $500 \mathrm{~nm}$ : a colored product was created in the reaction of 4-aminopyrine and hydrogen peroxide released from cholesterol. HDL-C concentration was determined colorimetrically at a wavelength of $500 \mathrm{~nm}$ after precipitation of other lipoproteins present in the studied biological material. Triacylglycerol (TG) concentration was determined colorimetrically at a wavelength of $520 \mathrm{~nm}$, after prior hydrolysis and the reaction of hydrogen peroxide, released from glycerol, with 4-chloro-phenol and 4-aminophenazone. All assays were performed with the appropriate kits from Alpha Diagnostics (Poland). LDL-C concentration was estimated using the Friedewald formula [12]. In addition, the ratios of TC/HDL-C and LDL-C/HDL-C were calculated.

All calculations were performed by means of Statistica v.6 (StatSoft, USA). Measurements were expressed as mean \pm SD. Normality of distribution was analyzed using the Shapiro-Wilk test. Significance of differences between individual pairs of mean values was evaluated using a two-way analysis of variance ANOVA (gender $\mathrm{x}$ activity) and a Tukey's post hoc test. The frequency of adverse lipid and lipoprotein concentrations was assessed using a Fisher's exact test. Differences were considered significant at $p<0.05$.

\section{Results}

As Table 1 clearly shows, all research participants were of a similar age. Women from the TW and NW groups had significantly lower body mass, body height,

Table 1. Anthropometric characteristics and weekly physical activity in women and men with different physical activity $($ mean $\pm \mathrm{SD})$

\begin{tabular}{lcccc}
\hline \multirow{2}{*}{ Variable } & \multicolumn{4}{c}{ Groups* $^{*}$} \\
& TW & TM & NW & NM \\
& $19.8 \pm 1.0$ & $20.6 \pm 1.2$ & $19.6 \pm 0.6$ & $19.8 \pm 0.9$ \\
\hline Age (years) & $169.0 \pm 5.5^{\mathrm{A}}$ & $182.1 \pm 7.5$ & $167.7 \pm 4.6^{\mathrm{B}}$ & $180.5 \pm 7.1$ \\
Body height $(\mathrm{cm})$ & $61.1 \pm 9.5^{\mathrm{A}}$ & $78.6 \pm 9.6$ & $58.4 \pm 6.0^{\mathrm{B}}$ & $75.4 \pm 8.1$ \\
Body mass (kg) & $21.6 \pm 3.7^{\mathrm{A}}$ & $23.6 \pm 2.1$ & $20.7 \pm 1.9^{\mathrm{B}}$ & $23.1 \pm 2.3$ \\
BMI & $22.5 \pm 3.7^{\mathrm{C}}$ & $12.3 \pm 2.5$ & $23.5 \pm 3.8^{\mathrm{D}}$ & $12.5 \pm 2.4$ \\
Body fat (\%) & $47.0 \pm 5.4^{\mathrm{A}, \mathrm{E}}$ & $68.8 \pm 7.8$ & $44.5 \pm 3.8^{\mathrm{B}}$ & $65.9 \pm 6.1$ \\
LBM & $8.4 \pm 2.5$ & $8.8 \pm 3.3$ & - & - \\
Training experience (years) & $11.2^{\mathrm{A}, \mathrm{F}}$ & $13.9^{\mathrm{D}}$ & 6.0 & 6.0 \\
Physical activity (h/week)** & & & & \\
\hline
\end{tabular}

*TW - training women, TM - training men, NW - non-training women, NM - non-training men; ${ }^{* *}$ Physical activity including physical education classes at the university and extracurricular trainings of endurance sports.

A Statistically significantly lower compared to the TM $(p<0.001)$; ${ }^{\text {B }}$ Statistically significantly lower compared to the NM $(p<0.001)$;

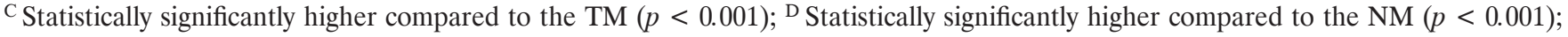

${ }^{\mathrm{E}}$ Statistically significantly higher compared to the NW $(p<0.05)$; ${ }^{\mathrm{F}}$ Statistically significantly higher compared to the NW $(p<0.001)$. 
BMI, LBM, as well as a significantly higher percentage of body fat compared to men from the TM and NM groups, respectively $(p<0.001)$. In addition, women from the TW group had a significantly higher LBM index than women from the NW group $(p<0.05)$. There were no statistically significant differences in the years of training between the TW group and the TM group. Compared to women from the NW group, women from the TW group were characterized by significantly higher levels of physical activity, expressed in hours/ week $(p<0.001)$, whereas men from the TM group had significantly higher physical activity, expressed in hours/week, in comparison to women from the TW group and men from the NM group $(p<0.001)$.

Table 2 presents data on the daily intake of energy, protein, fat, carbohydrates, fatty acids, and cholesterol. Women from the TW and NW groups had a significantly lower dietary intake of energy, total protein, and animal protein than the TM and NM groups, respectively $(p<0.001)$. Although the intake of protein per kilogram of body mass was significantly lower in the TW and NW groups compared to men from the TM and NM groups $(p<0.001)$, the percentage of energy from this nutrient was similar in all groups. Dietary intake of fat and percentage of energy derived from this nutrient in the TM and NM groups was significantly higher than in the TW and NW groups, respectively $(p<0.001)$. Men from the TM group had significantly higher carbohydrate intake in grams per day, as well as per kilogram of body mass in comparison with the NM and TW groups $(p<0.001)$. Dietary intake of this nutrient in the TW and NW groups was significantly lower than in the TM and NM groups $(p<0.001)$, while the percentage of energy derived from carbohydrates in the TW group was significantly higher than in the NW and TM groups $(p<0.001)$. Moreover, women from the TW and NW groups were characterized by a significantly lower intake of saturated and unsaturated fatty acids, as well as cholesterol, in comparison to the TM and NM groups, respectively $(p<0.001)$. At the same time, dietary intake of cholesterol in the TM group was significantly higher than in the TW and NM groups ( $p<0.001$ and $p<0.05$, respectively).

Pearson correlation coefficients between the dietary intake of cholesterol and fat, saturated fatty acids and animal pro tein in a diet are presented in Table 3. In all studied groups, the dietary intake of cholesterol was positively and significantly correlated with the intake of fat, animal protein and saturated fatty acids.

Pearson correlation coefficients between the percentage of body fat and plasma lipid profile are presented in Table 4. Only in the TM and NM groups was

Table 2. Daily intake of energy (kcal), protein, carbohydrates, fat, fatty acids ( $\mathrm{g}$ ) and cholesterol (mg) in women and men with different physical activity (mean $\pm \mathrm{SD})$

\begin{tabular}{lcccc}
\hline & & & Groups* \\
Parameter & TW & TM & NW & NM \\
& $(\mathrm{n}=29)$ & $(\mathrm{n}=30)$ & $(\mathrm{n}=30)$ & $(\mathrm{n}=27)$ \\
\hline Energy $(\mathrm{kcal})$ & $1855.9 \pm 618.0^{\mathrm{A}}$ & $3444.4 \pm 710.3^{\mathrm{C}}$ & $1828.8 \pm 554.4^{\mathrm{B}}$ & $2816.5 \pm 693.3$ \\
Total protein $(\mathrm{g})$ & $71.8 \pm 25.7^{\mathrm{A}}$ & $132.1 \pm 32.0$ & $71.2 \pm 21.3^{\mathrm{B}}$ & $116.2 \pm 33.4$ \\
& $\left(15.93 \pm 3.3^{* *}\right.$ & $(15.6 \pm 2.5)^{* *}$ & $(16.0 \pm 3.2)^{* *}$ & $(17.0 \pm 4.5)^{* *}$ \\
Protein/kg/day & $1.1 \pm 0.4^{\mathrm{A}}$ & $1.7 \pm 0.4$ & $1.2 \pm 0.3^{\mathrm{B}}$ & $1.5 \pm 0.4$ \\
Animal protein $(\mathrm{g})$ & $49.2 \pm 21.6^{\mathrm{A}}$ & $92.2 \pm 27.2$ & $49.1 \pm 17.9^{\mathrm{B}}$ & $82.6 \pm 29.4$ \\
Carbohydrates $(\mathrm{g})$ & $268.2 \pm 84.6^{\mathrm{A}}$ & $447.9 \pm 108.5^{\mathrm{C}}$ & $238.9 \pm 63.3^{\mathrm{B}}$ & $344.3 \pm 74.3$ \\
& $(55.9 \pm 7.4)^{* * \mathrm{E}}$ & $(50.1 \pm 6.3)^{* *}$ & $(50.6 \pm 8.3)^{* *}$ & $(47.7 \pm 5.5)^{* *}$ \\
Carbohydrates/kg/day & $4.5 \pm 1.6^{\mathrm{A}}$ & $5.7 \pm 1.5^{\mathrm{C}}$ & $4.1 \pm 1.0$ & $4.5 \pm 1.0$ \\
Fat (g) & $58.5 \pm 27.9^{\mathrm{A}}$ & $125.4 \pm 36.8$ & $68.1 \pm 32.6^{\mathrm{B}}$ & $110.4 \pm 44.0$ \\
Unsaturated fatty acids $(\mathrm{g})$ & $26.8 \pm 6.8)^{* * \mathrm{~A}}$ & $(32.3 \pm 6.9)^{* *}$ & $(31.7 \pm 7.9)^{* * \mathrm{~B}}$ & $(33.9 \pm 7.0)^{* *}$ \\
Saturated fatty acids $(\mathrm{g})$ & $28.6 \pm 14.4^{\mathrm{A}}$ & $62.9 \pm 21.2$ & $35.0 \pm 18.6^{\mathrm{B}}$ & $55.1 \pm 21.9$ \\
Cholesterol $(\mathrm{mg})$ & $25.3 \pm 12.5^{\mathrm{A}}$ & $53.9 \pm 17.5$ & $27.8 \pm 14.2^{\mathrm{B}}$ & $47.5 \pm 22.9$ \\
\hline
\end{tabular}

*See Table 1; **In parentheses - percentage of energy from the nutrient.

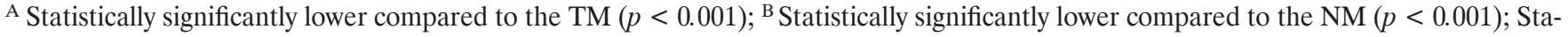
tistically significantly higher compared to the NM: ${ }^{\mathrm{C}} p<0.001 ;{ }^{\mathrm{D}} p<0.05$; ${ }^{\mathrm{E}}$ Statistically significantly higher compared to the NW and TM $(p<0.01)$. 
Table 3. Pearson correlation coefficients for cholesterol and fat, saturated fatty acids and animal protein in the groups of women and men with different physical activity

\begin{tabular}{lcccc}
\hline & & \multicolumn{2}{c}{ Groups* } \\
Parameter (g/day) & TW & TM & NW & NM \\
$(\mathrm{n}=29)$ & $(\mathrm{n}=30)$ & $0.69^{* * *}$ & $0.39^{* *}$ \\
\hline Fat & $0.66^{* * *}$ & $0.40^{* *}$ & $0.75^{* * *}$ & $0.39^{* * *}$ \\
Saturated fatty acids & $0.64^{* * *}$ & $0.59^{* * *}$ & $0.67^{* * *}$ & $0.54^{* *}$ \\
Animal protein & $0.48^{* * *}$ & $0.56^{* * *}$ & \\
\hline
\end{tabular}

* See Table $1 ; * * p<0.05 ; * * * p<0.001$.

Table 4. Pearson correlation coefficients for body fat percentage and plasma lipid profile in the groups of women and men with different physical activity

\begin{tabular}{|c|c|c|c|c|}
\hline \multirow[b]{2}{*}{ Parameter* } & \multicolumn{4}{|c|}{ Groups** } \\
\hline & $\begin{array}{c}\text { TW } \\
(\mathrm{n}=29)\end{array}$ & $\begin{array}{c}\text { TM } \\
(n=30)\end{array}$ & $\begin{array}{c}\text { NW } \\
(n=30)\end{array}$ & $\begin{array}{c}\text { NM } \\
(n=27)\end{array}$ \\
\hline $\mathrm{TG}(\mathrm{mmol} / \mathrm{L})$ & 0.32 & $0.61 * *$ & -0.27 & $0.53^{* * *}$ \\
\hline $\mathrm{TC}(\mathrm{mmol} / \mathrm{L})$ & 0.11 & 0.11 & 0.04 & 0.32 \\
\hline $\mathrm{HDL}-\mathrm{C}(\mathrm{mmol} / \mathrm{L})$ & -0.02 & -0.35 & 0.15 & 0.06 \\
\hline LDL-C (mmol/L) & 0.05 & 0.70 & 0.01 & 0.25 \\
\hline
\end{tabular}

* See Table $1 ; * * p<0.05$.

the percentage of body fat positively and significantly correlated with the plasma triglycerides concentration $(p<0.05)$. However, there were no significant relations between the percentage of body fat and other biochemical parameters, which constitute the components of lipid profile, in the studied groups.

Table 5 presents the assay results of the plasma lipid profiles in the groups. The concentrations of TC and HDL-C in the TW and NW groups were significantly higher than in the TM and NM groups, respectively $(p<0.05 ; p<0.001)$. However, there were no statistically significant differences between the studied groups in the concentrations of TG, LDL-C, and ratios of TC/ HDL-C and LDL-C/HDL-C.

Table 6 presents data on the frequency of adverse concentrations of TG, TC, LDL-C, and HDL-C in the groups. Adverse TG concentration in plasma was found in only 1 woman from the TW group. In the other groups, all participants had normal TG concentrations in plasma. Adverse TC concentration in

Table 5. Plasma lipid profile in women and men with different physical activity (mean \pm SD)

\begin{tabular}{lcccc}
\hline Parameter * & \multicolumn{2}{c}{ Groups** $^{*}$} & TW \\
& $(\mathrm{n}=29)$ & TM & NW & NM \\
& $0.7 \pm 0.3$ & $0.6 \pm 0.2$ & $0.6 \pm 0.1$ & $0.6 \pm 0.2$ \\
\hline TG $(\mathrm{mmol} / \mathrm{L})$ & $4.8 \pm 0.6^{\mathrm{A}}$ & $4.5 \pm 0.5$ & $4.7 \pm 0.7^{\mathrm{C}}$ & $4.3 \pm 0.6$ \\
TC $(\mathrm{mmol} / \mathrm{L})$ & $1.8 \pm 0.3^{\mathrm{B}}$ & $1.6 \pm 0.3$ & $1.8 \pm 0.3^{\mathrm{D}}$ & $1.6 \pm 0.3$ \\
HDL-C $(\mathrm{mmol} / \mathrm{L})$ & $2.7 \pm 0.5$ & $2.6 \pm 0.6$ & $2.6 \pm 0.6$ & $2.4 \pm 0.4$ \\
LDL-C $(\mathrm{mmol} / \mathrm{L})$ & $2.6 \pm 0.4$ & $2.9 \pm 0.8$ & $2.5 \pm 0.4$ & $2.7 \pm 0.4$ \\
TC/HDL-C & $1.5 \pm 0.4$ & $1.7 \pm 0.7$ & $1.4 \pm 0.4$ & $1.5 \pm 0.3$ \\
LDL-C/HDL-C & & & &
\end{tabular}

* TG - triglycerides; TC - total cholesterol; HDL-C - HDL cholesterol; LDL-C - LDL cholesterol; ** See Table 1.

Statistically significantly higher compared to the TM: ${ }^{\mathrm{A}} p<0.05 ;{ }^{\mathrm{B}} p<0.001$; Statistically significantly higher compared to the NM: ${ }^{\mathrm{C}} p<.05 ;{ }^{\mathrm{D}} p<0.001$. 
Table 6. Frequency of adverse concentrations of plasma TG, TC, LDL-C and HDL-C in women and men with different physical activity

\begin{tabular}{lcccc}
\hline Parameter & \multicolumn{4}{c}{ Groups* } \\
& $\begin{array}{c}\text { TW } \\
(\mathrm{n}=29)\end{array}$ & $\begin{array}{c}\text { TM } \\
(\mathrm{n}=30)\end{array}$ & $\begin{array}{c}\text { NW } \\
(\mathrm{n}=30)\end{array}$ & $\begin{array}{c}\text { NM } \\
(\mathrm{n}=27)\end{array}$ \\
\hline TG $(>1.7 \mathrm{mmol} / \mathrm{l})$ & $1 \mathrm{a}(3.5) \mathrm{b}$ & $0(0)$ & $0(0)$ & $0(0)$ \\
$\mathrm{TC}(>5.2 \mathrm{mmol} / \mathrm{l})$ & $7(24.1)$ & $5(16.7)$ & $9(30)$ & $2(7.4)$ \\
LDL $(>3.4 \mathrm{mmol} / \mathrm{l})$ & $3(10.3)$ & $4(13.4)$ & $4(13.4)$ & $1(3.7)$ \\
HDL $(<1.0 \mathrm{mmol} / \mathrm{l})$ & $0(0)$ & $0(0)$ & $0(0)$ & $0(0)$ \\
\hline
\end{tabular}

* See Table $1{ }^{\text {a }}$ no. of participants; ${ }^{b}$ group percentage.

plasma was observed in 7 women and 5 men among the training participants (the TW and TM, respectively) and 9 women and 2 men among the non-training participants (the NW and NM, respectively), whereas adverse LDL-C concentration in plasma was observed in 3 women and 4 men among the training participants and 4 women and 1 man among the non-training participants.

All studied individuals, regardless of their physical activity levels, had normal HDL-C concentrations in plasma. There were no significant differences in the frequency of adverse concentrations of lipids and lipoproteins in the groups.

\section{Discussion}

Women and men participating in the study differed in their body mass, body height, body fat percentage, BMI, and LBM due to their sex. The present study demonstrated that, compared to men, women had a significantly lower intake of energy, protein, carbohydrates and fat in terms of $\mathrm{g} /$ day and $\mathrm{g} / \mathrm{kg} / \mathrm{day}$, which is associated with a lower dietary intake of energy in daily food rations in this group.

Dietary intake of protein in the studied groups, per kilogram of body mass per day, did not significantly differ between the groups of men and women, both training and non-training. However, when taking into account the reference range for protein requirements based on sex and level of physical activity that was developed by Lemon [22], it can be concluded that the intake of this nutrient only in the group of non-training men was higher than the recommended values. Rossi et al. [34] found that the intake of this nutrient in the diet of young men practicing taekwondo was higher than the reference values. In the present study, dietary intake of carbohydrates, per kilogram of body mass per day, was similar in all the groups and met the recommendations for different physical activity levels. Only in the group of women with high physical activity was the intake of this nutrient slightly lower than the reference values [4]. According to the guidelines of the Third Report of the National Cholesterol Education Program (NCEP) Expert Panel [39] regarding the dietary intake of fat, the percentage of energy derived from this nutrient was only lower than the reference values in the diets of training women. Similar intakes of fat in the groups of young women and men with different levels of physical activity were also observed in the research by Malara et al. [26]. According to the guidelines of the Eurodiet project [19], the intake of saturated fats in all the groups in the present study was too high, as they constituted $11.5-14.5 \%$ of energy compared to the recommended intake of no more than $10 \%$ of energy. At the same time, the intake of unsaturated fats in the surveyed groups was slightly higher than the intake of saturated fats. In addition, it is worth noting that a dietary intake of cholesterol in the groups of men was high, and it far exceeded the recommended daily intake of $300 \mathrm{mg}$. Similarly, a high intake of cholesterol in a diet of young men was found by Głodek [13]. Research by McNamara [27] showed that consumption of cholesterol increased by $100 \mathrm{mg}$ adversely affects plasma lipid profile by raising TC concentration by $2.2-2.5 \mathrm{mg} / \mathrm{dl}$ and LDL-C concentration by $1.9 \mathrm{mg} / \mathrm{dl}$.

A significant amount of data in the literature indicate that the lipid profile of premenopausal women is more favorable as compared to the lipid profile of men of the same age, and it has a lower level of LDL-C and TG, and higher levels of HDL-C [5]. Women participating in the present study had a significantly higher concentration of HDL cholesterol in comparison with the corresponding groups of men. The concentrations of TG and LDL cholesterol did not significantly differ based on the sex of the participant. The plasma total cholesterol concentration in the groups of women was 
significantly higher than in the corresponding groups of men. A similar relationship in the groups of men and women with high physical activity was also found by Malara [26]; however, no such differences were observed in the groups of participants with low physical activity. On the basis of the data obtained in this study, it may be concluded that the differences in lipid profiles between women and men, observed regardless of their level of physical activity, were probably associated with sex. Furthermore, a significantly lower dietary intake of cholesterol with a concurrent higher concentration of cholesterol in plasma in the group of women in comparison to the group of men may indicate that the dietary intake of this nutrient only slightly affects the concentration of cholesterol in plasma. Lusis et al. [24] confirmed this assumption in their meta-analysis of the genetic basis of atherosclerosis.

In addition, significant amounts of data in the literature indicate that many young women excessively restrict the energy intake in their diets due to the fashion for being slim [9]. The consequences of energy shortages include abnormal sex hormone secretion, decreased bone density, and decreased insulin sensitivity as well as adverse changes in lipid profile [20]. In the present study, dietary intake of energy in both groups of women was lower than the recommended values [17], with the group of training women having a greater deficiency of energy in their diets compared with the non-training women. Therefore, it cannot be excluded that this deficiency had an adverse impact on the concentrations of total cholesterol and LDL cholesterol in plasma in both groups of women. On the other hand, the frequency of abnormal concentrations of these biochemical parameters in the group of non-training women was higher than in the group of training women. However, low credibility of selfreported dietary intakes [25] and the fact that in interviews women lower their dietary intakes of energy by $400-500 \mathrm{kcal} /$ day [31] suggest that the active participants of this study consumed more energy than reported in the interviews. This assumption is supported by the fact that the women in this group were characterized by proper body fat percentage.

In the present study, a trend toward an occurrence of adverse total cholesterol concentrations in the groups of women was higher than in the corresponding groups of men. At the same time, it was found that a higher percentage of women with abnormal concentrations of total cholesterol and LDL cholesterol in plasma were in the group of non-training women in comparison with the group of training participants, which may suggest a beneficial effect of physical exercise on the concentration of this biochemical parameter. On the other hand, a trend toward an occurrence of the abnormal concentrations of total cholesterol and LDL cholesterol in plasma was higher in the group of training men than in the group of non-training men, which can be associated with significantly higher dietary intakes of cholesterol in the group of training men. It cannot be excluded that a higher intake of fat and saturated fatty acids by men with high activity contributed to the observed differences, an assumption which is further supported by the research by Mente et al. [28] concerning the influence of diet on the development of coronary heart disease. Moreover, correlation coefficients obtained in the present study indicate that increased consumption of cholesterol in a diet is associated not only with an increased consumption of animal fats rich in saturated fatty acids, but also increased consumption of animal protein in foods.

In conclusion, increased physical activity only in the groups of men determined the increase in dietary intake of energy. There was no such relation in the groups of women. In addition, plasma lipid profile depended on sex, as evidenced by the similar concentrations of lipids and lipoproteins in both groups of women with different physical activity compared to the corresponding groups of men. Regardless of their levels of physical activity, women had a significantly higher concentration of total cholesterol in plasma, although their cholesterol intake was significantly lower than in the corresponding groups of men, which suggests that the concentration of cholesterol in plasma is largely dependent on the endogenous synthesis of this compound and that synthesis is only slightly intensified by the consumed cholesterol in a diet. At the same time, it is possible that a significantly higher intake of unsaturated fatty acids in the diets of men had an impact on a lower concentration of total cholesterol compared with the corresponding groups of women. The present study also found that despite the lack of statistically significant differences between the plasma lipid profile values within the groups of men and women with different physical activity, there was a trend toward a worse lipid profile in the training women and men in comparison with the corresponding groups of non-training participants. This may indicate that the increased physical activity was not a sufficient stimulus to induce positive changes in lipid profile. The present research does not allow for unequivocal assessment of this phenomenon. Yet in light of the results, it cannot be excluded that the observed differences are the result of a diet, as the dietary intake of cholesterol in the groups of training women and men was higher than in the corresponding groups of non-training participants. 


\section{References}

1. Bantle J.P., S.K. Raatz, W. Thomas, A. Georgopoulos (2000) Effects of dietary fructose on plasma lipids in healthy subjects. Am. J. Clin. Nutr., 72: 1128-1134.

2. Barona J., M.L. Fernandez (2012) Dietary cholesterol affects plasma lipid levels, the intravascular processing of lipoproteins and reverse cholesterol transport without increasing the risk for heart disease. Nutrients, 8: 1015-1025.

3. Bhardwaj S., S.J. Passi, A. Misra (2011) Overview of trans fatty acids: biochemistry and health effects. Diabetes. Metab. Syndr., 5: 161-164.

4. Burke L.M., B. Kiens, J.L. Ivy (2004) Carbohydrates and fat for training and recovery. J. Sports Sci., 22: 15-30.

5. Coelho V.G., L.F. Caetano, R. Liberatore Júnior Rdel, J.A. Cordeiro, D.R. Souza (2005) Lipid profile and risk factors for cardiovascular diseases in medicine students. Arq.Bras. Cardiol., 85: 57-62.

6. Durnin J.V., J. Womersley (1974) Body fat assessed from total body density and its estimation from skinfold thickness: measurements on 481 men and women aged from 16 to 72 years. Br. J. Nutr., 32: 77-97.

7. Durstine J.L., P.W. Grandjean, P.G. Davis, M.A. Ferguson, N.L. Alderson, K.D. DuBose (2001) Blood lipid and lipoprotein adaptations to exercise: a quantitative analysis. Sports Med., 31: 1033-1062.

8. Erkkila A.T., A.H. Lichtenstein (2006) Fiber and cardiovascular disease risk: how strong is the evidence? J.Cardiovasc.Nurs., 21: 3-8.

9. Fayet F., P. Petocz, S. Samman (2012) Prevalence and correlates of dieting in college women: a cross-sectional study. Int. J. Women's Health, 4: 405-411.

10. Freedman D.S., J.D. Otvos, E.J. Jeyarajah et al. (2004) Sex and age differences in lipoprotein subclasses measured by nuclear magnetic resonance spectroscopy: the Framingham Study. Clin. Chem., 50: 1189-1200.

11. Fried S.K., S.P. Rao (2003) Sugars, hypertriglyceridemia, and cardiovascular disease. Am. J. Clin. Nutr., 78: S873-S880.

12. Friedewald W.T., R. Levy, D. Fredrocson (1972) Estimation of concentration of low density lipoprotein concentration without use of the preparative ultracentrifugation. Clin. Chem., 18: 499-504.

13. Głodek E., M. Gil (2012) Evaluation of the nutrition model in students of university in Rzeszow. Rocz. Panstw. Zakl. Hig., 63: 313-317.

14. Habib S.S., M. Aslam, W. Hameed (2005) Gender differences in blood lipids and lipoprotein(a) levels in healthy individuals and patients with type 2 diabetes mellitus. Pak. J. Physiol., 1: 1-5.

15. Herzberg G.R. (2004) Aerobic exercise, lipoproteins, and cardiovascular disease: benefits and possible risks. Can. J. Appl. Physiol., 29: 800-807.
16. Hodson L., C.M. Skeaff, W.A. Chisholm (2001) The effect of replacing dietary saturated fat with polyunsaturated or monounsaturated fat on plasma lipids in free -living young adults. Eur. J. Clin. Nutr., 55: 908-915.

17. Human energy requirements (2004) Report of a Joint FAO/WHO/UNU Expert Consultation, FAO, Food and Nutrition Technical Report Series, No 1, FAO, Rome.

18. Jakobsen M.U., K. Overvad, J. Dyerberg, M. Schroll, B.L. Heitmann (2004) Dietary fat and risk of coronary heart disease: possible effect modification by gender and age. Am. J. Epidemiol., 160: 141-149.

19. Kafatos A., C.A. Codrington (1999) Nutrition and diet for healthy lifestyles in Europe: the 'Eurodiet' Project. Public. Health Nutr., 3A: 327-328.

20. Lawson E.A., K.K. Miller, V.A. Mathur, M. Misra, E. Meenaghan, D.B. Herzog, A. Klibanski (2007) Hormonal and nutritional effects on cardiovascular risk markers in young women. J. Clin. Endocrinol. Metab., 92: 3089-3094.

21. Lecerf J.M., M. de Lorgeril (2011) Dietary cholesterol: from physiology to cardiovascular risk. Br. J. Nutr., 106: 6-14.

22. Lemon P.W. (2000) Beyond the zone: protein needs of active individuals. J. Am. Coll. Nutr., 19: 513S-521S.

23. Li Z.L., J.D. Otvos, S. Lamon-Fava et al. (2003) Men and women differ in lipoprotein response to dietary saturated fat and cholesterol restriction. J. Nutr., 133: 3428-3433.

24. Lusis A.J., A.M. Fogelman, G.C. Fonarow (2004) Genetic basis of atherosclerosis: Part I. New genes and pathways. Circulation, 110: 1868-1873.

25. Lutosławska G., K. Mazurek, A. Kęska, A. Czajkowska, P. Żmijewski (2011) Validity of self-reported dietary intakes in young men and women with different physical activity. Med. Sportiva., 15: 119-124.

26. Malara M., G. Lutosławska_(2010) Physical activity, dietary habits and plasma lipoproteins in young men and women. Rocz. Panstw. Zakl. Hig., 61: 405-412.

27. McNamara D.J. (2000) Dietary cholesterol and atherosclerosis. Biochim. Biophys. Acta., 1529: 310-320.

28. Mente A., L. de Koning, H.S. Shannon, S.S. Anand (2009) A systematic review of the evidence supporting a causal link between dietary factors and coronary heart disease. Arch. Intern. Med., 169: 659-669.

29. Montoya M.T., A. Porres, S. Serrano et al. (2002) Fatty acid saturation of the diet and plasma lipid concentrations, lipoprotein particle concentrations, and cholesterol efflux capacity. Am. J. Clin. Nutr., 75: 484-491.

30. Müller H., A.S. Lindman, A.L. Brantsaeter, J.I. Pedersen (2003) The serum LDL/HDL cholesterol ratio is influenced more favorably by exchanging saturated with unsaturated fat than by reducing saturated fat in the diet of women. J. Nutr., 133: 78-83. 
31. Novotny J.A., W.V. Rumpler, H. Riddick et al. (2003) Personality characteristics as predictors of underreporting of energy intake on 24-hour dietary recall interviews. J. Am. Diet. Assoc., 103: 1146-1151.

32. Okura T., Y. Nakata, K. Tanaka (2003) Effects of exercise intensity on physical fitness and risk factors for coronary heart disease. Obes. Res., 11: 1131-1139.

33. Riccioni G., V. Sblendorio, E. Gemello et al. (2012) Dietary fibers and cardiometabolic diseases. Int. J. Mol. Sci., 13: 1524-1540.

34. Rossi L., R.E. Goya, M.A. Matayoshi, C.C. Cardoso Pereira, J. Bernardo da Silva (2009) Nutritional evaluation of taekwondo athletes. Braz. J. Biomotricity, 3: 159-166.

35. Sánchez-Muniz F.J. (2012) Dietary fibre and cardiovascular health. Nutr. Hosp., 27: 31-45.

36. Schubert C.M., N.L. Rogers, K.E. Remsberg et al. (2006) Lipids, lipoproteins, lifestyle, adiposity and fatfree mass during middle age: the Fels Longitudinal Study. Int. J. Obes., 30: 251-260.

37. Sonestedt E., N.C. Overby, D.E. Laaksonen, B.E. Birgisdottir (2012) Does high sugar consumption exacerbate cardiometabolic risk factors and increase the risk of type 2 diabetes and cardiovascular disease? Food. Nutr. Res., 56: 1-19.
38. Szponar L., K. Wolnicka, E. Rychlik (2000) Album fotografii produktów i potraw [Photo album of products and dishes]. Instytut Żywności i Żywienia, Warszawa.

39. The third report of the National Cholesterol Education Program (NCEP) expert panel on detection, evaluation and treatment of high cholesterol in adults (Adult treatment panel III). Circulation. 2002, 106: 3143-421.

40. Vidon C., P. Boucher, A. Cachefo, O. Peroni, F. Diraison, M. Beylot (2001) Effects of isoenergetic high-carbohydrate compared with high-fat diets on human cholesterol synthesis and expression of key regulatory genes of cholesterol metabolism. Am. J. Clin. Nutr., 73: 878-884.

\section{Received 19.08.2013 \\ Accepted 9.12.2013}

(C) University of Physical Education, Warsaw, Poland

\section{Acknowledgements}

Research funded by the Ministry of Science and Higher Education (DM-2) conducted at the Józef Piłsudski University of Physical Education in Warsaw, Poland 